\title{
Clinical Profile and Results in Cancers Treated with Nivolumab: A Single Centre Study
}

\author{
B. J. Srinivasa, Bhanu Prakash Lalkota, Sulav Sapkota, Vinu Sarathy, Nithin Bayas, \\ Radheshyam Naik
}

Department of Medical Oncology, HCG Cancer Specialty Center, Bangalore, India

Email: sripav77@yahoo.co.in

How to cite this paper: Srinivasa, B.J., Lalkota, B.P., Sapkota, S., Sarathy, V., Bayas, N. and Naik, R. (2018) Clinical Profile and Results in Cancers Treated with Nivolumab: A Single Centre Study. Open Journal of Immunology, 8, 107-111. https://doi.org/10.4236/oji.2018.84007

Received: November 18, 2018 Accepted: December 26, 2018 Published: December 29, 2018

Copyright $\odot 2018$ by author(s) and Scientific Research Publishing Inc. This work is licensed under the Creative Commons Attribution International License (CC BY 4.0).

http://creativecommons.org/licenses/by/4.0/

\begin{abstract}
Introduction: Immunotherapy is also called as biologic therapy or biotherapy. Immunotherapy is quickly becoming an important component in the multipronged approaches being developed to treat certain forms of cancer. The goal of immunotherapy is to strengthen the body's own natural ability to combat cancer by enhancing the effectiveness of the immune system. This study tries to compile response rates and side effect profile of Nivolumab. Methods: Patients with performance status of 3 or less than 3, who failed on 2 or more lines of therapy except melanoma and have received minimum 3 cycles of Nivolumab were taken in to this study. Study subjects followed for minimum of 3 cycles for assessment. Each patient received $240 \mathrm{mg}$ of Nivolumab once in 15 days for 3 - 6 cycles. Results: The median age of 20 patients was 53 years ( $26-79 \mathrm{yrs}) .18$ patients were male and 2 patients were female. Progressive disease (PD) was observed in 12 patients with immunotherapy treatment and partial response (PR) was observed in 3 patients, and stable disease (SD) in 5 patients. Conclusion: This is one of the Indian study mainly reporting experiences with Nivolumab. Large sample size is required to conclude about the efficacy of the study. Nivolumab is more tolerated in Indian patients compared to other reported studies.
\end{abstract}

\section{Keywords}

Cancer, Immunotherapy, Nivolumab

\section{Introduction}

The global cancer burden is expected to nearly double to 21.4 million cases and 13.2 million deaths by 2030 [1]. Consistently cancer is treated by the different practice like surgery, radiation and chemotherapy. When in early 1900 Dr Wil- 
liam Coley started using immunotherapy in cancer where it is well founded in Asthma and Arthritis. One hallmark of cancer is immune evasion, in which the immune system does not mount an effective antitumor response [2]. Immunotherapy is also called as biologic therapy or biotherapy. Immunotherapy is quickly becoming an important component in the multipronged approaches being developed to treat certain forms of cancer. The landscape of cancer therapy has been recently transformed by the emergence of immunotherapy involving the targeting of immune checkpoints. The goal of immunotherapy is to strengthen the body's own natural ability to combat cancer by enhancing the effectiveness of the immune system [3]. There are three known main categories of immunotherapy: immune response modifiers (cytokines), monoclonal antibodies and vaccines [4]. Nivolumab (ONO-4538/BMS-936558/MDX-1106) is a fully human IgG4 monoclonal antibody that targets programmed cell death-1 (PD-1), one of the T-cell surface membrane receptors. Nivolumab received the world's first regulatory approval in Japan in July 2014 for the treatment of unresectable malignant melanoma. Nivolumab is also undergoing development for the treatment of other indications based on checkmate trails [5]. Nivolumab is approved by Food and Drug Administration (FDA) for Melanoma Non-Small Cell Lung Cancer (NSCLC), Renal Cell Carcinoma (RCC), Hodgkin Lymphoma, Head and Neck Cancer, Urothelial Carcinoma, Colorectal Cancer, and Hepatocellular Carcinoma (HCC) and approved with combination in other indications [6]. The maximal tolerated dose (MTD) for nivolumab has not been identified, and a similar safety profile has been demonstrated across tumour types and dose levels $(0.1-10 \mathrm{mg} / \mathrm{kg}$ ). More recently FDA both $3 \mathrm{mg} / \mathrm{kg}$ nivolumab and $240 \mathrm{mg}$ fixed dose are based on pharmacokinetic data [7]. This study explores response rates and side effect profile of Nivolumabin a single centre.

\section{Methods}

In this study, a total of 20 eligible patients who received Nivolumab in HCG Cancer Speciality Center from 2017 to March 2018 were prospectively analysed.

Inclusion Criteria: Patients with performance status of 3 or less than 3 , failed on 2 or more lines of therapy except melanoma and have received minimum 3 cycles of Nivolumab were taken in to this study. Patients who had financial toxicity were excluded from this study. Study subjects observed minimum of $3 \mathrm{cy}-$ cles for assessment with PETCT and based on response followed till progression or death. Microsatellite instability (MSI) and Programme Death Ligand (PD-L1) expression was not tested in these entire patients. Standard Response Evaluation Criteria in Solid Tumors (V.1.1) [8] criteria for response assessment and CTCAE 4.0 version for toxicity assessment is used for analysis. Base line clinical examination, TSH, serum amylase, RBS, LFT, and CBC were done and monitored before each cycle of treatment. CT/PET-CT/MRI is used for response assessment before and after treatment. Each patient received $240 \mathrm{mg}$ of Nivolumab once in 15 days for $3-6$ cycles. All the patients were followed until progression or death 
due to any cause.

Primary objective of this study was to assess the response rate of Nivolumab and evaluation of toxicity was secondary objectives.

\section{Results}

There were total 20 patients were included for the analysis and all patients were able to complete minimum 3 - 4 cycles of Immunotherapy.

The median age of 20 patients was 53 years ( 26 - 79 yrs). 18 patients were male and 2 patients were female. Immunotherapy is given as $1^{\text {st }}$ line in 6 patients and $\geq 2$ lines in 14 patients. With regards to safety, all of our patients are well tolerated with immunotherapy except for grade 1/grade 2 anaemia/thrombocytopenia in 6 patients. Progressive disease (PD) was observed in 12 patients with immunotherapy treatment and partial response (PR) were observed in 3 patients, stable disease (SD) in 5 patients (Table 1 ).

\section{Discussion}

Immunotherapy is an advanced treatment for metastatic cancer, where it mainly involves with the body's natural immune response to cancer and secondly it helps the immune system to find and kill cancer cells. In this era it has shown lot of promise to increase overall response and survival of the patients. This study explores immunotherapy response in advanced and metastatic cancer patients in

Table 1. Baseline demographic and clinical characteristics of patients received Nivolumab.

\begin{tabular}{|c|c|}
\hline Patient Characteristics & Whole group \\
\hline AGE & $53(26-79$ yrs $)$ \\
\hline \multicolumn{2}{|l|}{ SEX } \\
\hline Male & $18(90 \%)$ \\
\hline Female & $2(10 \%)$ \\
\hline \multicolumn{2}{|l|}{ ECOG (PS) } \\
\hline $0-1$ & $14(70 \%)$ \\
\hline$>2$ & $6(30 \%)$ \\
\hline \multicolumn{2}{|l|}{ Metastatic Sites } \\
\hline 1 & $7(35 \%)$ \\
\hline$\geq 2$ & $13(65 \%)$ \\
\hline \multicolumn{2}{|l|}{ Line of Immunotherapy } \\
\hline $1^{\text {st }}$ line & $6(30 \%)$ \\
\hline$\geq 2$ line & $14(70 \%)$ \\
\hline \multicolumn{2}{|l|}{ ADRs with Immunotherapy } \\
\hline No toxicity & 14 \\
\hline Toxicity & 6 (Anaemia) \\
\hline AVG Duration of Immunotherapy & (3- 6 cycles $)$ \\
\hline \multicolumn{2}{|l|}{ Response rate ( 3 - 6 cycles) } \\
\hline PR & 3 \\
\hline $\mathrm{SD}$ & 5 \\
\hline $\mathrm{PD}$ & 12 \\
\hline
\end{tabular}


single centre. All six patients with metastatic melanoma received first line treatment with immunotherapy and 2 patient had SD (20\%) and 4 had PD, whereas checkmate trail 037 trail used this drug in $2^{\text {nd }}$ line and had $32 \%$ of response rate [7]. In the 4 HCC patients, 2 patients progressed and 2 patients achieved stable disease, while in checkmate 40 trail, it has shown $15 \%$ overall response [8]. Out of 4 patients with NSCLC only 1 patient had PR, other 3 patients were progressed. Whereas checkmate 057 trails, there was 19\% responses rate [9]. Out of 3 carcinoma bladder patients, 1 patient obtained PR, other patients were achieved SD [1] and PD [1], and there was 19.6\% response in checkmate 275 trails [10]. In head \& neck patients out of 3 only 1 patient got partial response and 1 patient acquire stable disease, in checkmate 141 trail it was detected 13.3\% [11]. As response rate varies from $15 \%$ to $30 \%$ in various cancers as per the immunotherapy trails, patients in this study also had similar response rates except in HCC. Small sample size in individual cancer is limiting factor in this study.

Most of our patient had only grade $1-2$ fatigue (60\%), where as it was around $12 \%-24 \%$ in various single studies [12]. There was no grade III-IV adverse events observed in the study and no patient were discontinued on treatment due to side effects. In various FDA approved trails for Nivolumab grade III toxicity was $12 \%-69 \%$ and grade IV was $7 \%-20 \%$ [13] [14].

This is one of the Indian study mainly reporting experiences with Nivolumab. Large sample size is required to conclude about the efficacy of the study. Nivolumab is more tolerated in Indian patients compare to other reported studies.

\section{Conflicts of Interest}

The authors declare no conflicts of interest regarding the publication of this paper.

\section{References}

[1] Jemal, A., Bray, F., Center, M.M., Ferlay, J., Ward, E. and Forman, D. (2011) Global Cancer Statistics. CA: A Cancer Journal for Clinicians, 61, 69-90.

https://doi.org/10.3322/caac.20107

[2] Hanahan, D. and Weinberg, R.A. (2011) Hallmarks of Cancer: The Next Generation. Cell, 144, 646-674. https://doi.org/10.1016/j.cell.2011.02.013

[3] de Pillis, L.G., Gu, W. and Radunskaya, A.E. (2006) Mixed Immunotherapy and Chemotherapy of Tumors: Modeling, Applications and Biological Interpretations. Journal of Theoretical Biology, 238, 841-862. https://doi.org/10.1016/j.jtbi.2005.06.037

[4] Rosenbaum, E. and Rosenbaum, I. (2005) Everyone's Guide to Cancer Supportive Care: A Comprehensive Handbook for Patients and Their Families. Andrews McMeel Publishing, Kanas City.

[5] Topalian, S.L., Hodi, F.S., Brahmer, J.R., et al. (2012) Safety Activity, and Immune Correlates of Anti-PD-1 Antibody in Cancer. The New England Journal of Medicine, 366, 2443-2454. https://doi.org/10.1056/NEJMoa1200690

[6] https://www.accessdata.fda.gov/drugsatfda_docs/label/2017/125554s055lbl.pdf 
[7] Yoo, S.H., Keam, B., Kim, M., Kim, S.H., Kim, Y.J., Kim, T.M., Kim, D.W., Lee, J.S. and Heo, D.S. (2018) Low-Dose Nivolumab Can Be Effective in Non-Small Cell Lung Cancer: Alternative Option for Financial Toxicity. ESMO Open, 3, e000332.

[8] Eisenhauer, E.A., Therasse, P., Bogaerts, J., et al. (2009) New Response Evaluation Criteria in Solid Tumours: Revised RECIST Guideline (Version 1.1). European Journal of Cancer, 45, 228-247. https://doi.org/10.1016/j.ejca.2008.10.026

[9] Weber, J.S., et al. (2015) Nivolumab versus Chemotherapy in Patients with Advanced Melanoma Who Progressed after Anti-CTLA-4 Treatment (CheckMate 037): A Randomised, Controlled, Open-Label, Phase 3 Trial. The Lancet Oncology, 16, 375-384. https://doi.org/10.1016/S1470-2045(15)70076-8

[10] El-Khoueiry, A.B., Sangro, B., Yau, T., et al. (2017) Nivolumab in Patients with Advanced Hepatocellular Carcinoma (CheckMate 040): An Open-Label, Non-Comparative, Phase 1/2 Dose Escalation and Expansion Trial. The Lancet, 389, 2492-2502. https://doi.org/10.1016/S0140-6736(17)31046-2

[11] Paz-Ares, L., Horn, L., Borghaei, H., Spigel, D.R., et al. (2015) Phase III, Randomized Trial Trial (CheckMate 057) of Nivolumab (NIVO) versus Docetaxel (DOC) in Advanced Non-Squamous Cell (Non-SQ) Non-Small Cell Lung Cancer (NSCLC). The New England Journal of Medicine, 373, 1627-163.

[12] Sharma, P., Retz, M., Siefker-Radtke, A., et al. (2017) Nivolumab in Metastatic urothelial Carcinoma after Platinum Therapy (CheckMate 275): A Multicentre, Single-arm, Phase 2 Trial. The Lancet Oncology, 18, 312-322. https://doi.org/10.1016/S1470-2045(17)30065-7

[13] Ferris, R.L., Blumenschein Jr., G., Fayette, J., et al. (2016) Nivolumab for Recurrent Squamous-Cell Carcinoma of the Head and Neck. The New England Journal of Medicine, 375, 1856-1867. https://doi.org/10.1056/NEJMoa1602252

[14] Naidoo, J., Page, D.B., Li, B.T., et al. (2015) Toxicities of the Anti-PD-1 and Andti-PD-L1 Immune Checkpoint Antibodies. Annals of Oncology, 26, 2375-2391. 